Cahiers d'études japonaises

\title{
La représentation du nu féminin dans l'art contemporain japonais : recherche d'une identité esthétique, culturelle et sociale
}

Mathilde Collet

\author{
(2) OpenEdition \\ Journals \\ Édition électronique \\ URL : https://journals.openedition.org/cipango/1620 \\ DOI : $10.4000 /$ cipango.1620 \\ ISSN : 2260-7706 \\ Éditeur \\ INALCO \\ Édition imprimée \\ Date de publication : 1 juin 2011 \\ Pagination : 288-292 \\ ISBN : 978-2-85831-195-8 \\ ISSN : 1164-5857
}

Référence électronique

Mathilde Collet, "La représentation du nu féminin dans l'art contemporain japonais : recherche d'une identité esthétique, culturelle et sociale », Cipango [En ligne], 18|2011, mis en ligne le 23 septembre 2013, consulté le 30 juin 2021. URL : http://journals.openedition.org/cipango/1620 ; DOI : https:// doi.org/10.4000/cipango.1620

Ce document a été généré automatiquement le 30 juin 2021.

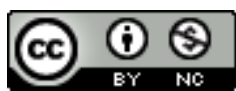

Cipango est mis à disposition selon les termes de la Licence Creative Commons Attribution - Pas d'Utilisation Commerciale 4.0 International. 


\title{
La représentation du nu féminin dans l'art contemporain japonais : recherche d'une identité esthétique, culturelle et sociale
}

\author{
Mathilde Collet
}

\section{RÉFÉRENCE}

Mathilde COLLET, La représentation du nu féminin dans l'art contemporain japonais : recherche d'une identité esthétique, culturelle et sociale, mémoire de Master 2, sous la direction de Christian Galan et Tomomi Ota, Université de Toulouse II - Le Mirail, 2012.

Reflet de l'ambivalence de la modernité japonaise, l'art contemporain des années 1980 à nos jours s'impose dans la multiplicité de ses formes comme autant de réponses à la quête d'une identité japonaise moderne, qui débuta à l'ère Meiji, et se poursuivit jusqu'aux récents bouleversements identitaires où il est question d'une nouvelle perception des genres dans la société contemporaine. Le nu féminin artistique apparaît, au regard des grands évènements socio-historiques japonais du $\mathrm{xx}^{e}$ siècle, comme un élément indissociable de l'évolution de la perception de l'identité japonaise. Les artistes se sont effectivement investis, et continuent de s'investir dans cette quête d'identité au travers de leurs créations, recherchant l'affirmation de l'individu en lui-même tout aussi bien que celle d'un peuple entier dans l'art. Pouvoir suggestif et symbolique, beauté des formes, capacité d'objectivation du corps, d'abstraction des idées... Dans l'histoire de l'art japonais, où n'existe pas de longue tradition esthétique du nu, le corps nu féminin reste beaucoup plus inspirant et plus apte à devenir le symbole d'idées nouvelles que le corps masculin, s'affirmant comme un support visuel parfait pour illustrer la recherche identitaire japonaise. Les travaux de certains artistes contemporains sont ainsi à l'image des interrogations et des métamorphoses de la 
société japonaise de ces trente dernières années, et apportent des réponses, sinon des pistes de réflexion, à la question de la quête d'identité inhérente à l'art japonais. Leurs œuvres recouvrent divers supports tels que la peinture, la photographie, l'installation, la performance, la danse et la création numérique.

Bien ancrées dans la réalité contemporaine, il est pourtant révélateur de voir à quel point les œuvres de certains artistes se réfèrent aux périodes de la guerre et de l'aprèsguerre dans un objectif critique envers la société actuelle. On peut effectivement retrouver dans la défaite de la Seconde Guerre, l'occupation américaine et le boom économique les facteurs historiques, culturels et sociaux à l'origine des pratiques artistiques contemporaines dans lesquelles s'imbrique la question du corps féminin contemporain liée aux enjeux de la quête d'identité. Les artistes s'approprient ainsi le média du corps nu féminin comme une forme de résistance de la culture visuelle aux conséquences de la modernisation occidentale. Dans cette optique, le nu féminin devient le support de va-et-vient esthétiques et sémantiques entre Histoire passée et contemporanéité, entre techniques traditionnelles et concepts modernes, et témoigne du désir des artistes d'inscrire leur art dans une temporalité affiliée à leur histoire personnelle ou à celle de leur pays, dans laquelle ils viennent puiser inspiration et force. Murakami Takashi 村上隆 (1965-) et Aida Makoto 会田誠 (1962-), sont ainsi deux artistes représentatifs par leur technique, qui intègre de nouveaux outils comme la création numérique dans leur processus de réalisation, mais marque par l'utilisation des techniques picturales nihonga leur volonté d'un retour formel aux racines artistiques japonaises. L'artiste polyvalent Morimura Yasumasa 森村泰昌 (1951-), dans ses reprises de célèbres tableaux de l'Histoire de l'Art occidentaux, créé par le syncrétisme artistique des interfaces de suspension du temps, de l'espace et du soi. Il propose une représentation de la perte des essences dans la complexité d'un monde contemporain postmodernisé, où les identités individuelles, culturelles, nationales, en viennent à se démultiplier et à se flexibiliser tels des rôles cinématographiques éphémères.

3 Le corps nu féminin peut également devenir l'expression dissidente de revendications contre les tabous de la société contemporaine. Qu'il s'agisse d'obscénité, de tabous culturels reposant sur des questions de morale, de ségrégation, les artistes les plus audacieux et touchés intimement par ces considérations décident de faire entendre leurs voix, et c'est dans les supports les plus suggestifs que leurs images percutantes viennent se déplier sous le regard, exposant dans le corps nu féminin ce qui habituellement reste caché. Censure des poils pubiens, jugements sur l'homosexualité, rigidité des conceptions de genres, désinformation sur le sujet du sida, amnésie historique sur une partie de l'histoire de la prostitution... Les artistes s'attaquent à des critères préétablis et rigides définissant des identités sociales auxquelles ils ne se sentent pas appartenir, et viennent transgresser dans leur art ces identités préconçues. Morimura Yasumasa et Takano Ryūdai 鷹野隆大 (1963-), abordent le thème des corps travestis et transsexuels, démontrant dans leurs photographies l'instabilité des genres. Le collectif Dumb Type ダム・タイプ secoue les a priori sur l'homosexualité et le sujet du sida dans des représentations hétéroclites de danse et de performance, à l'esthétique ultra-contemporaine, tandis que Bubu de la Madeleine ブブ・ド・ラ・マ ドレーヌ (dates inconnues) et Shimada Yoshiko 嶋田美子 (1959-) reconsidèrent dans une installation photographique et vidéo le statut de la prostitution sous un angle historique se voulant féministe. Les tabous persistants à apposer le sceau du silence sur 
des aspects révélateurs de la sexualité japonaise contemporaine se brisent le temps de manifestations percutantes qui placent l'authenticité au centre du pouvoir de persuasion.

4 Depuis les années 1990, un glissement esthétique s'est opéré par rapport à la représentation $\mathrm{du} \mathrm{nu}$ au long $\mathrm{du} \mathrm{Xx}^{\mathrm{e}}$ siècle : à la représentation des corps de femmes, beaucoup d'artistes préfèrent le corps gracile des jeunes filles, et les chairs tendent à incarner la figure de la shōjo, jeune fille japonaise consommatrice et narcissiquement auto-identifiée à ce qui relève du kawaii, de l'innocence, du pathos. Cependant, nombre de représentations, dans l'art contemporain, vont plus loin que ces assertions, et viennent abattre par des procédés artistiques critiques les normes et les comportements que le genre féminin se voit contraint d'adopter. L'étude de la représentation du corps féminin chez les artistes féminines telles que Aoshima Chiho 青島千穂 (1974-), Takano Aya タ力ノ綾 (1976-), Yanagi Miwa やなぎみわ (1967-), Nagashima Yurie 長島有里枝 (1973-), permet de comprendre de quelle façon, depuis les années 1980, celles-ci s'émancipent des productions masculines et s'imposent sur la scène artistique; et comment elles font de leur art un moyen de réflexion sur la prise en main de leur image, de leur corps et de leur avenir, sur les genres, et sur la place de la femme dans la société. Cette mise en lumière du corps féminin par les artistes femmes appuie non seulement une reconquête de son propre corps par la femme, mais également l'affirmation d'une subjectivité féminine qui s'impose dans la société, au détriment des repères masculins de la nouvelle génération, lesquels s'étiolent doucement depuis que les années 1990 ont discrédité les valeurs et les codes hégémoniques symbolisés par la culture et l'économie patriarcale.

Le corps féminin, auparavant peint et dépeint majoritairement par les hommes et pour les hommes, aussi bien dans les arts que dans les autres champs de la société, devient un leitmotiv de l'art contemporain féminin. Non plus au service d'une vision exclusivement masculine, le corps de la femme se déploie au gré de la sensibilité et de la subjectivité d'artistes femmes, comme s'il incarnait l'enjeu d'une nouvelle affirmation subjective au sein de la société japonaise qui passerait par la réappropriation de l'image de leur propre corps par les femmes. Accompagnant cette nouvelle prise de pouvoir, les statuts passent d'un idéal rigide de féminité et de masculinité à une perception plus souple des codes de genre. En n'hésitant plus à s'approprier des techniques spécifiques, les femmes affirment leurs points de vue sur le monde et les genres, tout comme elles commencent à conquérir artistiquement le corps nu des hommes en exemple de cette souveraineté nouvelle dans l'art. Parallèlement à cette évolution, les hommes, qu'il s'agisse d'artistes tels que Morimura Yasumasa ou Takano Ryūdai, de modèles d'artistes femmes qui travaillent sur le nu masculin telles qu'Ishiuchi Miyako 石内都 (1947-) ou Nomura Sakiko 野村佐紀子 (1967-), ou simplement d'une tranche de la jeune génération qui participe de ce que les sociologues appellent la " féminisation de la société ", semblent perdre certains de leurs repères de masculinité et de virilité pour glisser vers un genre masculin alternatif, qui semble annoncer un effacement progressif des frontières et des codes stricts entre les genres au profit d'identités nouvelles. 